\title{
Temporal Patterning of Saccadic Eye Movement Signals
}

\author{
Daniel L. Kimmel and Tirin Moore \\ Department of Neurobiology, Stanford University School of Medicine, Stanford, California 94305
}

Electrical microstimulation is used widely in experimental neurophysiology to examine causal links between specific brain areas and their behavioral functions and is used clinically to treat neurological and psychiatric disorders in patients. Typically, microstimulation is applied to local brain regions as a train of equally spaced current pulses. We were interested in the sensitivity of a neural circuit to a train of variably spaced pulses, as is observed in physiological spike trains. We compared the effect of fixed, decelerating, accelerating, and randomly varying microstimulation patterns on the likelihood and metrics of eye movements evoked from the frontal eye field of monkeys, while holding the mean interpulse interval constant. Our results demonstrate that the pattern of microstimulation pulses strongly influences the probability of evoking a saccade, as well as the metrics of the saccades themselves. Specifically, the pattern most closely resembling physiological spike trains (accelerating pattern) was most effective at evoking a saccade, three times more so than the least effective decelerating pattern. A saccade-triggered average of effective random trains confirmed the positive relationship between accelerating rate and efficacy. These results have important implications for the use of electrical microstimulation in both experimental and clinical settings and suggest a means to study the role of temporal pattern in the encoding of behavioral and cognitive functions.

Key words: motor control; neural coding; electrical stimulation; saccade; temporal coding; oculomotor

\section{Introduction}

Electrical microstimulation of neural tissue has been used widely to examine causal links between activity of specific brain areas and a behavioral function. For instance, since its introduction in the 19th century by Fritsch and Hitzig (1870), experimentalists have used microstimulation to link frontal cortical areas to skeletomotor (Penfield and Jasper, 1954; Graziano et al., 2002) and oculomotor control (Ferrier, 1886; Robinson and Fuchs, 1969; Bruce et al., 1985); occipital and temporal cortical areas to visual perception (Brindley and Lewin, 1968; Salzman et al., 1990); temporal cortical areas to object recognition (Penfield and Jasper, 1954; Afraz et al., 2006); parietal cortical areas to tactile perception (Romo et al., 1998); subcortical areas to oculomotor control (Robinson, 1972; Sparks et al., 1987); and even to link certain areas to cognitive functions, such as attention (Moore and Fallah, 2001, 2004; Moore and Armstrong, 2003; Cavanaugh and Wurtz, 2004; Muller et al., 2005), decision-making (Gold and Shadlen, 2003; Hanks et al., 2006), and learning and reward (Rolls, 1975; Williams and Eskandar, 2006). In addition, clinicians have more recently used electrical stimulation of the CNS [deep brain stimulation (DBS)] in the treatment of neurological and psychiatric disorders in patients (Perlmutter and Mink, 2006).

In the experimental setting, microstimulation mimics natural neurophysiology to a sufficient extent that its effects are function-

Received Jan. 29, 2007; revised May 5, 2007; accepted May 30, 2007.

This work was supported by National Institutes of Health Grant EY14924, the Pew Charitable Trust, and the Sloan Foundation. D.L.K. was supported by a Stanford Bio-X Graduate Fellowship and the Medical Scientist Training Program. We thank D. S. Aldrich for technical assistance, R. J. Schafer for experimental assistance, and K. M. Armstrong, S. Hestrin, V. Mante, and J. B. Reppas for valuable suggestions on this manuscript.

Correspondence should be addressed to Daniel L. Kimmel, Department of Neurobiology, Stanford University School of Medicine, Stanford, CA 94305. E-mail: dkimmel@stanford.edu.

DOI:10.1523/JNEUROSCI.0386-07.2007

Copyright $\odot 2007$ Society for Neuroscience $\quad$ 0270-6474/07/277619-12\$15.00/0 ally and anatomically specific to a given brain area (e.g., Salzman et al., 1990; Tolias et al., 2005), and evoked responses are within the range of physiological behavior (e.g., Graziano et al., 2002). These qualities have enabled the technique to move beyond merely relating a brain area with a behavioral function and to link the observed spatial and temporal dynamics of neuronal activity to their functional significance: for instance, linking the volume of excited tissue to the fidelity of the motion signal in visual area MT (Murasugi et al., 1993); linking the rate of microstimulation to the perceived frequency of tactile vibration (Romo et al., 1998); and linking the phase of simultaneous microstimulation trains to the summing or averaging of saccadic vectors (Brecht et al., 2004).

Despite the ability of microstimulation to examine these and other signal properties, microstimulation has not tested the relevance of the temporal variability observed in most physiological spike trains. For most cortical neurons, the distribution of interspike intervals (ISIs) is probabilistic (Shadlen and Newsome, 1998), and the average ISI changes reliably in relation to a sensory or motor event, e.g., a decrease in ISI preceding an eye movement (Sparks, 1978; Bruce and Goldberg, 1985; Hanes and Schall, 1996). Nevertheless, nearly all applications of microstimulation have used a fixed interval between consecutive microstimulation pulses. Indeed, microstimulation has been criticized for not emulating natural spiking activity (Strick, 2002).

We asked whether microstimulation might be used to determine the behavioral significance of the temporal patterning of inputs. We tested the sensitivity of the oculomotor system to the pattern of microstimulation pulses delivered to the frontal eye field (FEF), an area of frontal cortex that encodes fixed-vector saccadic eye movements that can be evoked with microstimulation (Bruce et al., 1985; Russo and Bruce, 1993). The probability of evoking saccades from the FEF increases with increasing stim- 
ulation current, frequency, and train duration (Tehovnik and Sommer, 1997). The FEF served as a promising candidate site in which to test temporal pattern sensitivity because of previous work suggesting that the oculomotor system differentiates saccade specification and execution signals by their stimulation frequency (Sparks, 1978; Glimcher and Sparks, 1993) and evidence that the short-term dynamics in the FEF before a saccade play a role in saccade timing (Hanes and Schall, 1996). Therefore, differential changes in frequency within a microstimulation signal may be important to and discriminated by the oculomotor system.

We compared the effect of fixed (FIXED), decelerating (DECEL), accelerating (ACCEL), and randomly varying microstimulation patterns on the likelihood and metrics of evoked saccades while holding the mean interpulse interval (IPI) constant. Our results demonstrate that the pattern of microstimulation pulses strongly influences the probability of evoking a saccade, as well as the metrics of the saccades themselves. Specifically, the pattern most closely resembling physiological spike trains (accelerating pattern) was most effective at evoking a saccade, three times more so than the least effective decelerating pattern. A saccade-triggered average of effective random trains confirmed the positive relationship between accelerating rate and efficacy. The results are consistent with a model of saccade generation in which a lowfrequency "primer" signal contributes to saccade specification and likelihood, a high-frequency "trigger" signal then initiates the movement, and a subsequent "driver" signal modifies the movement.

\section{Materials and Methods}

Behavioral task. We trained two rhesus monkeys (Macaca mulatta) to fixate a central fixation point (FP) displayed against a black background on an LCD video monitor $(52 \times 87 \mathrm{~cm}, 60 \mathrm{~Hz}$; NEC Display Solutions, Itasca, IL) in an otherwise dimly lit room. The monkey's eye position was measured by way of a scleral search coil and stored at a 200 or $500 \mathrm{~Hz}$ sampling frequency while the head remained fixed. Each behavioral trial began with the appearance of the FP. The monkey was required to maintain fixation within a $2^{\circ}$ diameter electronic window for a variable period of time (451-950 ms). On approximately half of the trials (55.7\%), we applied electrical microstimulation via a microelectrode to the FEF 105 ms after extinguishing the FP. On a portion of these trials, the stimulation was sufficient to evoke a saccade along a predictable vector. We held constant the electrical current throughout a block of trials while we varied the pattern of current pulses, selecting one of five patterns for each trial, thus totaling five stimulation conditions. The remaining half of the trials lacked stimulation and was divided into two conditions. Because the trains of stimulation were preceded by the audible switching of a relay, we included this switching but without stimulation in $25 \%$ of the nonstimulation trials (SWITCH-NO STIM). The remaining $75 \%$ of nonstimulation trials had neither switching nor stimulation (NO STIM). At the end of each trial, the monkey was rewarded with a drop of juice for having maintained fixation during the required period (i.e., FP illuminated), regardless of whether a saccade was subsequently evoked. The next trial began after a $2 \mathrm{~s}$ delay.

Trial conditions were randomly interleaved across all stimulation and nonstimulation conditions. A block of trials consisted of several repetitions (4-12) of each condition. The NO sTIM condition occurred three times more frequently than the other conditions, which otherwise occurred with equal frequency. At each FEF stimulation site, we ran four to six blocks, each block using a different level of electrical stimulation current. All task events, including visual stimuli, eye position tracking, reward, and triggering of individual microstimulation pulses were controlled by custom code written for the CORTEX state system software (http://www.cortex.salk.edu).

Electrical microstimulation of the FEF. Electrical microstimulation consisted of a $35 \mathrm{~ms}$ train of biphasic constant-current pulses (cathodal leading, $0.25 \mathrm{~ms}$ pulse width per phase) delivered with a Grass (West Warwick, RI) stimulator (S88) and two Grass stimulation isolation units (PSIU-6). The biphasic pulse ensured that charge would not accumulate on the electrode, which might interfere with subsequent pulses or damage neural tissue (Tehovnik, 1996). Current amplitude was measured via the voltage drop across a $1 \mathrm{k} \Omega$ resistor in series with the return lead of the current source. We verified that the current delivered remained constant for all pulses within a train. All stimulation was delivered via varnishcoated tungsten microelectrodes of $0.2-1.0 \mathrm{M} \Omega$ impedance (measured at $1 \mathrm{kHz})$.

In each monkey, the FEF was first localized on the basis of its surrounding physiological and anatomical landmarks and the ability to evoke fixed-vector saccades on one-half of the trials with stimulation at currents of $<50 \mu \mathrm{A}$ (Bruce et al., 1985). This microstimulation criterion was achieved in a separate calibration task run before each experiment. In the calibration task, we delivered a $100 \mathrm{~ms}$ stimulation train of equally spaced biphasic current pulses $(200 \mathrm{~Hz})$. We used these trials to confirm that the evoked saccades were fixed-vector, to obtain an estimate of the threshold current $(<50 \mu \mathrm{A}$ criterion) required to evoke a saccade on one-half the trials, and to establish the evoked saccadic vector of the FEF site. When using the shorter train durations of the experimental protocol (35 ms), we frequently required higher stimulation currents $(>50 \mu \mathrm{A})$ to achieve efficacies at and $>50 \%$.

After an FEF site had been identified, we began the experimental protocol of delivering the pulse patterns described below. Stimulation current was held constant throughout a block of trials. Generally, for the first block, we used the threshold current determined in the calibration task. We then selected three to five additional currents so as to span the range of currents from those that were completely ineffective to those that were $100 \%$ effective for all pulse patterns. At times, it was not feasible to find currents that fulfilled this later case because some pulse patterns were so ineffective that the required currents would have been inordinately high.

Pulse patterns. We used five pulse patterns, four of which had eight pulses delivered over $35 \mathrm{~ms}$, making for a common mean IPI of $5 \mathrm{~ms}$, and one additional random pattern (Fig. 1A). (1) FIXED, in which the IPI remained constant at $5 \mathrm{~ms}$ throughout the train. (2) DECEL, in which the IPI became progressively longer throughout the train according to $i=1$ $+n$, where $i$ is the IPI in milliseconds, and $n$ is the IPI position, constrained between 1 and 7 , inclusive. Thus, the IPIs for DECEL in order were $2,3,4,5,6,7,8$ ms. (3) ACCEL, in which the IPI became progressively shorter throughout the train according to $i=9-n$. Thus, ACCEL was symmetrical with DECEL, containing the same IPIs in exactly the opposite order: 8, 7, 6, 5, 4, 3, 2 ms. (4) Random-order pattern (RO), in which the order of IPIs used in ACCEL and DECEL was randomly shuffled. (5) Random-interval pattern (RI), in which eight pulses were assigned randomly to 2 -ms-wide bins with 1 pulse per bin. Because the $35 \mathrm{~ms}$ time window could not be divided evenly into 2 -ms-wide bins, one-half of the RI trains contained 17 bins ( $34 \mathrm{~ms}$ train), and one-half contained 18 bins (36 ms train). Train lengths were randomly interleaved. RI trains could deliver eight pulses in less than, but not more than, $35 \mathrm{~ms}$, and therefore their mean IPI could be slightly less than the mean of $5 \mathrm{~ms}$ common to the other pulse patterns.

The accelerating discharge of FEF visuomotor cells can begin as early as 200-300 ms before a saccade (Bruce and Goldberg, 1985). Previous microstimulation studies have used train durations from 12 to $400 \mathrm{~ms}$ (Bruce et al., 1985; Russo and Bruce, 1993; Tehovnik and Lee, 1993; Tehovnik and Sommer, 1997). We selected the present train duration so as to address the distinction between sensitivity to the temporal pattern of pulses and sensitivity to the time-varying average rate of pulses for which the average is taken over some portion $(d t)$ of the total train duration. Although our present study does not explicitly distinguish these two possibilities, it does put an upper limit on any possible values of $d t$ as less than the total train duration. We reasoned that at the lower limit of $d t$, the two possibilities converge (i.e., pattern sensitivity $=$ sensitivity to changes in the rate averaged spike-to-spike). In addition, most accounts in the literature that implicitly support the time-varying average rate possibility (by averaging neural activity over some time window) do so with values of $d t$ in the range of 100 s to 1000 s of milliseconds. We were therefore motivated to minimize the train duration and thereby mini- 
mize possible values of $d t$. However, it has been found empirically that to evoke saccades from the FEF, microstimulation train durations must be at least $12-25 \mathrm{~ms}$ even when using much higher frequencies $(900 \mathrm{~Hz})$ and currents $(400 \mu \mathrm{A})$ than used presently (Tehovnik and Sommer, 1997). We used the train duration of $35 \mathrm{~ms}$ to reconcile our goals of evoking saccades and minimizing train duration.

Saccade detection. Stimulation-evoked saccades were detected by four criteria: speed, direction, latency, and amplitude. Off-line for each trial, the mean displacement $5-55 \mathrm{~ms}$ before microstimulation was subtracted from the eye position data for the entire trial, thereby centering each saccade start point at $[0,0]$. The horizontal and vertical components of the eye position data were converted into their polar counterparts of amplitude $r$ and direction $\theta$. Eye speed $s$ as function of time $t$ was then computed as the derivative of $r(t)$. All values of $s(t)$ equal to or exceeding the speed threshold $S$ were scored as saccades. Criterion $S$ was determined separately for each monkey (ultimately, both monkeys were fitted best by $S=35 \%$ s) and is consistent with published minimum velocities for saccades (Becker, 1989). Consecutive values of $s(t)$ meeting $S$ were treated as part of the same saccade. In addition, nonconsecutive values of $s(t)$ meeting $S$ and occurring within $30 \mathrm{~ms}$ of each other were grouped together as a single saccade, as were any intervening values of $s(t)$ that may have failed the criterion $S$. This grouping made the criterion $S$ less susceptible to high-frequency noise in $s(t)$ and is based on the empirical minimum for intersaccade intervals (Becker, 1989).

After initially detecting saccades using the speed criterion, the criteria of direction, latency, and amplitude were applied to select for saccades evoked by stimulation. The saccade start and stop positions were used to compute the overall saccadic vector, from which we extracted final saccade direction $\theta_{\mathrm{f}}$ and amplitude $r_{\mathrm{f}}$. Latency was defined as the saccade start time relative to time of microstimulation onset (stimulation trials) or to time when stimulation would have been delivered for SWITCH-NO STIM. Saccades were scored as "stimulation-evoked" when their direction fell within $\pm 45^{\circ}$, inclusive, of the prototypical direction $D$, their latency was less than or equal to time $T$, and their amplitude was greater than or equal to the threshold amplitude $A$. Criterion $D$ was defined as the modal direction of all saccades detected in stimulation trials for the given site. Criterion $T$ was chosen as $100 \mathrm{~ms}$ after microstimulation onset. Stimulation-evoked saccades tended to have very little variation in direction or latency compared with voluntary saccades, which tended to fall well outside of the criteria $D$ and $T$. Criterion $A$ was set at $1^{\circ}$ of visual angle so as to reduce the rate of false-positive detections attributable to high-frequency line noise in the eye position signal. Occasionally, this would result in a false negative for a very short amplitude saccade, but these errors were more acceptable than false positives in that they would not confound our subsequent analyses of the saccade metrics.

The saccade detection algorithm was applied to the NO STIM trials as well. Because in these trials no event marked the onset of microstimulation, we chose to detect saccades within the window 100-200 ms after fixation as a control measure of false positives.

Analysis. For a given FEF site and stimulation current, we determined the proportion of trials in which a saccade was evoked for each condition separately. This proportion is termed the "saccade probability" and applies to analyses of both the block of trials as a whole and to individual saccades from within that block. The relationship of saccade probability to absolute stimulation current was fit with a sigmoid function for each condition at each FEF site. From these fits, we derived the "threshold current" for each condition, defined as the stimulation current at which a saccade was evoked on half of the trials. The "site threshold" was defined as the threshold current for FIXED.

Absolute stimulation currents were transformed into normalized values so that data from currents across FEF sites could be grouped. Specifically, each stimulation current was classified into one of seven "ordinal current" groups based on the relationship of the current to site threshold. The stimulation current immediately less than site threshold was classified as ordinal current " 3 ." The stimulation current just below ordinal current 3 would become ordinal current " 2 " and so on; the same system applies to currents successively greater than site threshold becoming ordinal currents "4," "5," "6," and "7." Importantly, the same assignment of stimulation current to ordinal current was made for all pulse patterns within an FEF site. For example, all trials from FEF site 9 (Fig. 1) using 45 $\mu \mathrm{A}$ stimulation current are classified as ordinal current 3 , regardless of pulse pattern. The system of ordinal current normalization served to group currents of similar saccade-evoking efficacy across all FEF sites.

The regressions of saccade amplitude on saccade latency were fit with a rational function (i.e., quotient of two polynomials) of the following form:

$$
A=\frac{p_{1}(-L)+p_{2}}{q_{1}-L},
$$

where $A$ and $L$ are log normalized saccade amplitude and latency, respectively, and $p_{1}, p_{2}$, and $q_{1}$ are constants least-square fit for each pulse pattern. The " $(-L)$ " notation serves to emphasize that the function saturates with increasingly negative values of log normalized latency. A rational function was chosen because it appeared to fit the data well and because a one-tailed saturating function appealed to our predictions as to the effects of very short and very long latencies on amplitude (see Discussion).

\section{Results}

We studied the influence of microstimulation pulse pattern on saccades evoked from the FEF of two monkeys performing a fixation task. At each of 26 FEF sites, we applied five types of microstimulation trains, which differed in the pattern of current pulses delivered in $35 \mathrm{~ms}$. Each pattern consisted of eight pulses, in which the IPI remained constant, increased, decreased, or varied randomly (Fig. 1A). We refer to these respective patterns in terms of their frequency profiles, namely FIXED, DECEL, ACCEL, and two random profiles. For FIXED, the IPI was held constant at $5 \mathrm{~ms}$. For DECEL, ACCEL, and one random pattern, RO, the set of IPIs was held constant, ranging from 2 to $8 \mathrm{~ms}$ with a common mean IPI of $5 \mathrm{~ms}$. In the RO pattern, the interval order was shuffled randomly from trial-to-trial. In the second random pattern, RI, pulses were assigned with equal probability to 2 -ms-wide bins with one pulse per bin. To maximize the space of possible patterns, RI was allowed to terminate short of $35 \mathrm{~ms}$, and thus the mean IPI was allowed to fall slightly $<5 \mathrm{~ms}$. We applied the above patterns, one chosen randomly during each behavioral trial, and examined the efficacy of each in evoking saccades, as well as their influence on the evoked saccade metrics.

\section{Example FEF site and analysis}

Results from an example FEF site are shown in Figure $1 B-G$. At this site, saccades to the lower contralateral visual field were evoked with currents from 45 to $65 \mu \mathrm{A}$ (Fig. $1 B$ ). The current at which the probability of evoking a saccade was $50 \%$ using the FIXED pulse pattern was $53 \mu \mathrm{A}$ (site threshold). The efficacy of the remaining four patterns differed not only from FIXED, but also from one another (Fig. 1C). To evoke saccades with $50 \%$ probability, ACCEL required the lowest stimulation current $(44 \mu \mathrm{A})$, DECEL the highest current $(59 \mu \mathrm{A})$, and the random patterns (RO and RI) higher currents than FIXED, although approximately equal to each other ( 56 and $55 \mu \mathrm{A}$, respectively). Note that at the site threshold current, there was a sixfold difference in efficacy between ACCEL and DECEL. Overall, the random patterns showed less sensitivity to changes in current than the other patterns.

In addition to the efficacy of evoking saccades, we examined the influence of pulse pattern on the metrics of the evoked saccades (Fig. 1D-G). Specifically, we measured latency, amplitude, peak velocity, and duration. In choosing which saccades to compare, one could compare those evoked with different pulse patterns at the same stimulation current. However, as suggested in Figure $1 C$ (and borne out in the population data), pulse pattern influences stimulation efficacy independent of stimulation cur- 
rent. Therefore, any differences in saccade metrics between pulse patterns at the same stimulation current may not be attributable to a direct effect of pulse pattern, but instead to differences in stimulation efficacy. Thus, we attempted to equalize the stimulus parameters between conditions by comparing saccades evoked with the same stimulation efficacy or saccade probability. Individual saccades were assigned a value of saccade probability based on their corresponding pulse pattern and block of trials in which they were evoked (see Materials and Methods). We then regressed each metric on saccade probability.

For saccades evoked with FIXED at the example site, all metrics were significantly correlated with saccade probability (latency, $r=-0.69, p<0.002$; amplitude, $r=0.73, p<10^{-3}$; peak velocity, $r=0.76$, $p<10^{-3}$; duration, $r=0.64, p<0.005$ ). In addition, saccades evoked with ACCEL at a given saccade probability appeared to have the longest latencies, the smallest amplitudes, the slowest peak velocities, and the shortest durations. In contrast, saccades evoked with DECEL appeared to have the shortest latencies, the largest amplitudes, the fastest peak velocities, and the longest durations (regressions not shown).

\section{Saccade probability}

At 14 FEF sites, we were able to test a sufficient number of stimulating currents (4-6) to measure the efficacy of each pulse pattern and to evoke enough saccades (43119 per site) to compare their metrics. For each site, we determined site threshold using the FIXED pattern (median, $47 \mu \mathrm{A}$ ) and classified each stimulation current into one of seven ordinal currents based on its value relative to site threshold (see Materials and Methods). Across all sites, we computed the proportion of saccade-evoking trials at a given ordinal current and pulse pattern (Fig. 2A). The resulting functions confirmed the effect of pulse pattern on saccade probability that we observed for the example site: the lowest ordinal current threshold was obtained with ACCEL, the highest with DECEL, and intermediate thresholds were obtained with FIXED, RO, and RI (ordered from low to high). The ordinal current normalization produced sigmoidal curves that, for FIXED, crossed the point of 0.5 saccade probability between ordinal currents 3 and 4 (vertical line), which represents the position of raw site thresholds relative to which ordinal currents were assigned, thus confirming that the normalization maintained the relative distribution of raw currents used within each site.

We observed that, for all pulse patterns, saccade probability varied over the span of ordinal currents 3-5, whereas lower ordinal currents never evoked a saccade, and higher currents always evoked a saccade. In addition to representing the dynamic range of ordinal current, these middling currents were also the most consistently sampled across sites $(\sim 100$ trials per ordinal current
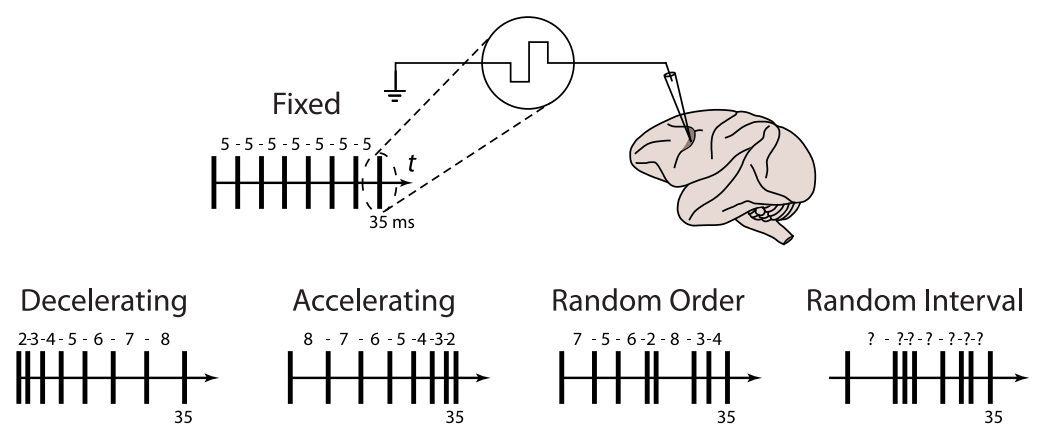

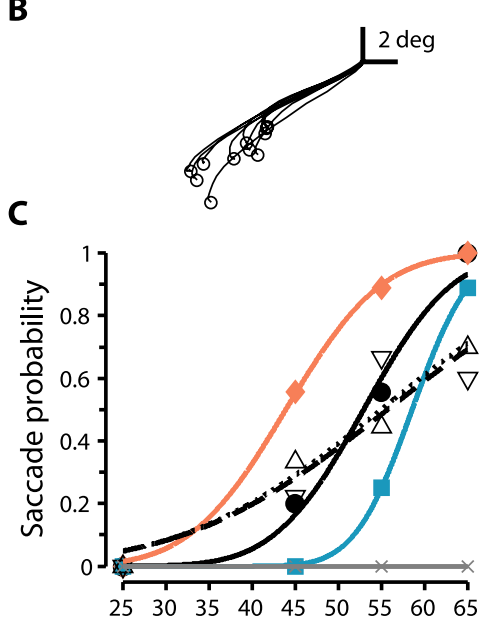

Figure 1. Effects of pulse pattern on saccades from an example FEF site. $\boldsymbol{A}$, Five patterns of electrical microstimulation current pulses were delivered to the FEF (shaded region). Each vertical bar represents a biphasic current pulse (enlarged in inset); the horizontal lines represent time $(t)$ increasing from left to right and with the first pulse at $t=0$. Individual IPIs are shown above (the final random train excepted). The pulse patterns depicted are (top-bottom, left-right) as follows: FIXED (constant IPI), DECEL (linearly decreasing IPI symmetrically opposite of DECEL), RO (same IPIs as DECEL and ACCEL but in random IPIS). $\boldsymbol{B}-\mathbf{G}$, Data and example analysis from a single FEF site are shown. $\boldsymbol{B}$, Raw traces of saccade trajectory evoked at a suprathreshold stimulation current $(65 \mu \mathrm{A})$ using the FIXED pulse pattern are plotted. Open circles indicate saccade endpoints. $\boldsymbol{C}$, The saccade probability is plotted against the stimulation current for each pulse pattern. $\boldsymbol{D}-\boldsymbol{G}$, For each stimulation-evoked saccade, atency $(\boldsymbol{D})$, amplitude $(\boldsymbol{E})$, peak velocity $(\boldsymbol{F})$, and duration $(\boldsymbol{G})$ of individual evoked saccades are plotted against saccade probability of the pulse pattern as obtained for the block of trials in which the saccades were evoked. Lines are shown for significant $(p<0.05)$ linear regressions. per pulse pattern) and thus provided the most reliable estimates of saccade probability. We therefore focused all subsequent analyses of pulse-pattern efficacy and saccade metrics on trials at these middling currents. Figure $2 B$ shows the proportion of saccadeevoking trials as a function of pulse pattern collapsed across all sites and across the three middling currents.

Pulse pattern significantly affected the percentage of trials in which saccades were evoked $\left(\chi^{2}=191, \mathrm{df}=4, p<10^{-16}\right)$, with ACCEL proving most effective (78\%), followed by FIXED (64\%), RO (50\%), RI (43\%), and finally DECEL as the least effective (27\%). Thus, there was a threefold difference in efficacy between the most (ACCEL) and least (DECEL) effective pulse patterns. Moreover, post hoc pairwise comparisons yielded significant differences in saccade probability between all pairs of pulse patterns $(p<0.001$ by Bonferroni-corrected $\chi^{2}$ ), except for the two random ones $(p>0.07)$. The data from individual monkeys followed the same trend (Fig. 2 B, gray symbols), and thus all analyses were collapsed 
FIXED
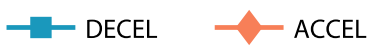

$-\mathbf{\Lambda} \cdot \mathrm{RO}$

' $\nabla \cdot$

A

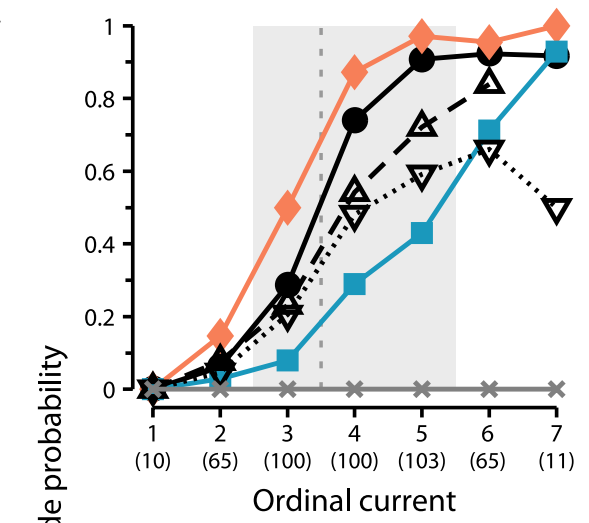

C

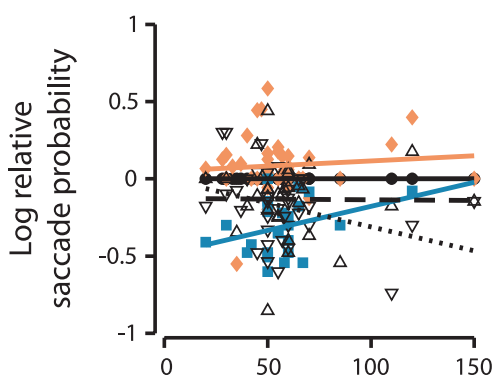

Stimulation current $(\mu \mathrm{A})$

D

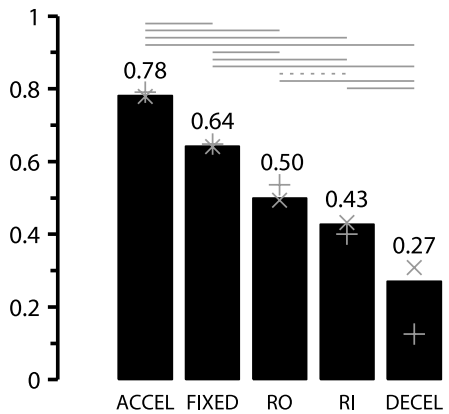

Pulse pattern
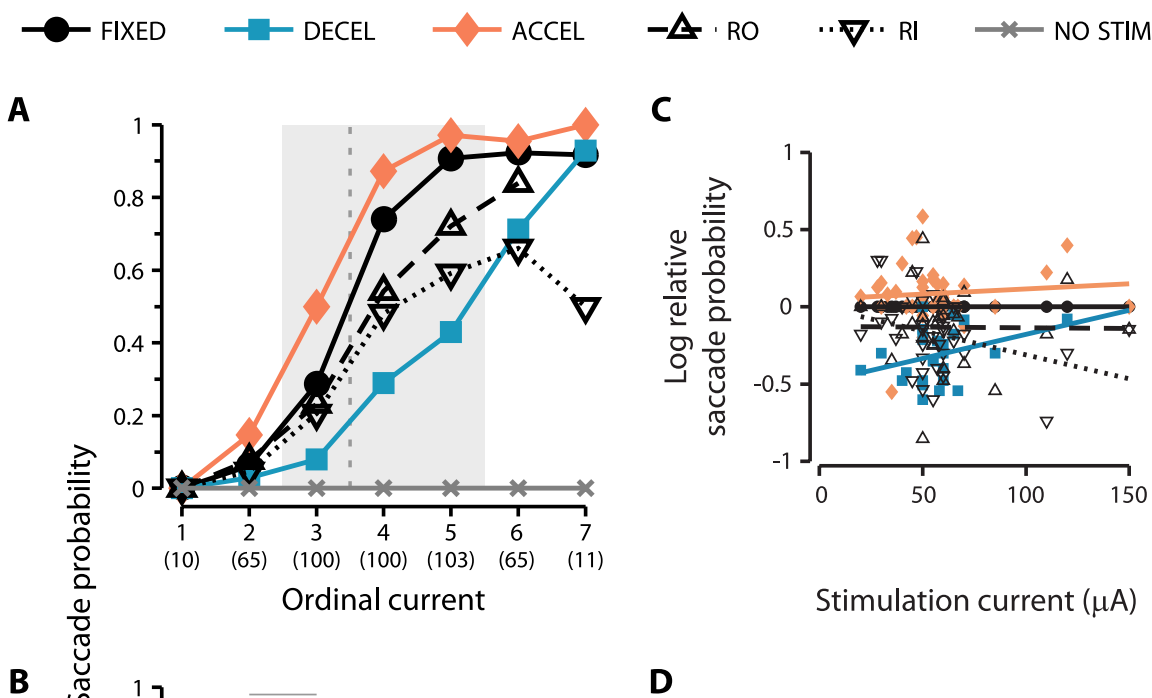

the "main sequence" (Becker, 1989; Chen, 2006; Knight and Fuchs, 2006; our unpublished observations). We confirmed that the evoked saccades followed this main sequence (Fig. 2D). Specifically, saccade peak velocity was significantly linearly correlated $\left(p<10^{-16}\right)$ with amplitude for each pulse pattern with slopes ranging from $47 \mathrm{~s}^{-1}$ for DECEL to $63 \mathrm{~s}^{-1}$ for ACCEL (regression values are listed in Table S1, available at www.jneurosci.org as supplemental material).

Although all evoked saccades fell along a main sequence, we found pulse-patterndependent differences in the exact relationship of peak velocity to amplitude (ANCOVA, $F=3.22$, $\mathrm{df}=4, p<0.05$ ). For the same amplitude movement, DECEL saccades had significantly slower peak velocities than those of FIXED, ACCEL, and RO $\left(p<10^{-3}\right.$, Bonferroni corrected). In addition, RI produced significantly slower peak velocities at the same amplitude than FIXED and ACCEL $(p<0.05$, Bonferroni corrected).

As for the example FEF site, we analyzed the influence of pulse pattern on saccade latency, amplitude, peak velocity, and duration as a function of saccade probability. We performed two analyses, both of which were designed to control for the intrinsic site-to-site variability of evoked saccade metrics (Bruce et al., 1985). (1) For statistical comparisons, we used an ANCOVA model that included as factors pulse pattern, probability of saccade, and stimulation site. (2) For purposes of display, we normalized the saccade metrics by site (each raw saccade value was divided by the mean value of all ACCEL saccades at that site) and regressed the log normalized values on the saccade probability of the pulse pattern as obtained for the block of trials in which the saccades were evoked (Fig. 3)

across both monkeys. No saccades were detected in the two nonstimulation conditions, NO STIM and SWITCH-NO STIM.

We tested whether the effect of pulse pattern held constant across a range of stimulation currents. For each block of trials, we normalized the saccade probability of each pulse pattern to that of the FIXED pattern and regressed the normalized values on the stimulation current of that block (Fig. 2C). There was no significant correlation $(p>0.05)$ between normalized saccade probability and stimulation current for any pulse pattern, indicating that the effect of pulse pattern was consistent across the range of middling currents tested $(20-150 \mu \mathrm{A})$. The laddering of regression lines from ACCEL (highest relative saccade probability) to DECEL (lowest) recapitulates the main effect of pulse pattern on efficacy observed in the ordinal current analysis.

\section{Saccade metrics}

Voluntary and stimulation-evoked saccades share a strong relationship between peak velocity and amplitude (which is quasilinear over the range of amplitudes obtained presently) known as (see Materials and Methods)

Using the statistical model, we found significant effects of pulse pattern, saccade probability, and stimulation site on all saccade metrics (see Table S2A, available at www.jneurosci.org as supplemental material). In particular, saccades evoked with DECEL, although less common on average, tended to have lower latencies, larger amplitudes, faster peak velocities, and longer durations than saccades of equal probability evoked by every other pulse pattern. Conversely, saccades evoked with ACCEL tended to have the highest latencies, shortest amplitudes, slowest peak velocities, and shortest durations (all pairwise comparisons are listed in Table S2B, available at www.jneurosci.org as supplemental material). It was not surprising that the sign and degree of the effects of a given pulse pattern on amplitude, peak velocity, and duration were similar given the strong mutual correlations between these metrics discussed above as the main sequence (Fig. 2D) (Becker, 1989). Finally, whereas DECEL saccades traveled farther, reached faster speeds, and did so over more time than ACCEL saccades at the same saccade probability, recall that the main 
A

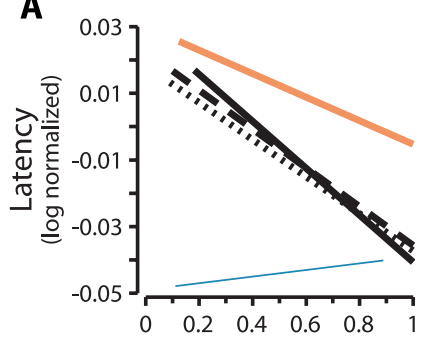

B

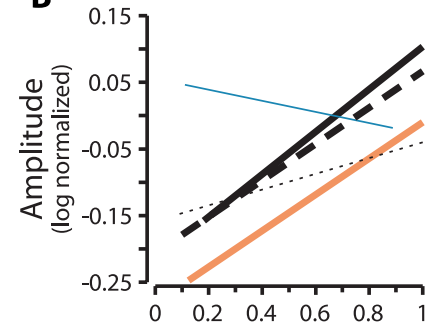

C
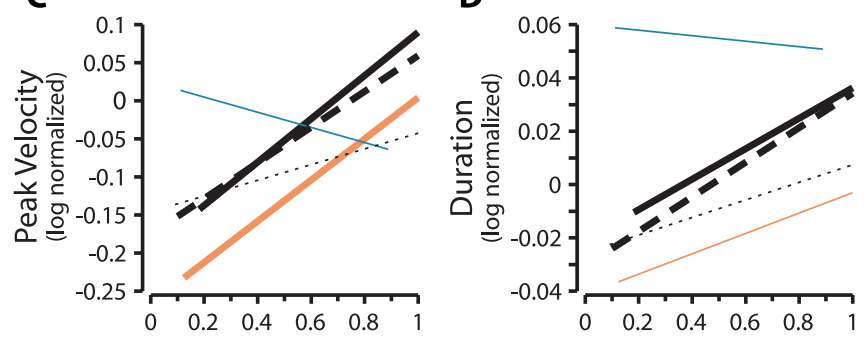

Saccade probability

Figure 3. Effect of pulse pattern on saccade metrics. $\boldsymbol{A}-\boldsymbol{D}$, Four metrics are analyzed for saccades evoked at the three middling currents across all FEF sites: latency $(\boldsymbol{A})$, amplitude $(\boldsymbol{B})$, peak velocity $(\boldsymbol{C})$, and duration $(\boldsymbol{D})$. Plotted separately for each pulse pattern are linear regressions of log normalized values for single saccades on the saccade probability of the pulse pattern as obtained for the block of trials in which the saccades were evoked. Thick lines represent significant $(p<0.05)$ regressions.

sequences obtained for these pulse patterns showed that ACCEL saccades were nonetheless faster than DECEL saccades for the same amplitude movement (Fig. 2D).

The regressions of normalized metrics on saccade probability showed, in general, that an increasing saccade probability correlated with a lower latency, greater amplitude, faster peak velocity, and longer duration. These correlations were significant $(p<$ 0.05 ) in at least one if not all four metrics for all pulse patterns; DECEL was the notable exception, showing no relationship between any metric and saccade probability (all regression values and histograms of saccade probability are presented in Table S3 and Fig. S1, respectively, available at www.jneurosci.org as supplemental material). The significant correlations are not surprising given that both saccade metrics and the likelihood of evoking a saccade share a common dependence on stimulation current (Tehovnik and Sommer, 1997; our unpublished observations). However, this dependence alone did not account for the effect of pulse pattern on saccade metrics, as confirmed by a separate analysis that showed a significant effect of pulse pattern on saccade metrics independent of stimulation current and stimulation site (ANCOVA, $p<10^{-6}$ for all metrics) (see Table S4, available at www.jneurosci.org as supplemental material). That is, the greater current required by, say, DECEL to achieve efficacy comparable to ACCEL did not explain the larger amplitude saccades evoked by DECEL at the same saccade probability. Even at the same stimulation current, saccades evoked with the different pulse patterns differed in their metrics (see Fig. S2, available at www.jneurosci.org as supplemental material).

We noted that the pulse pattern evoking the shortest average latency saccades (i.e., DECEL) also evoked the greatest average amplitude, peak velocity, and duration saccades; the opposite was true for ACCEL saccades. We were interested in whether this relationship between latency and amplitude held for individual sac-
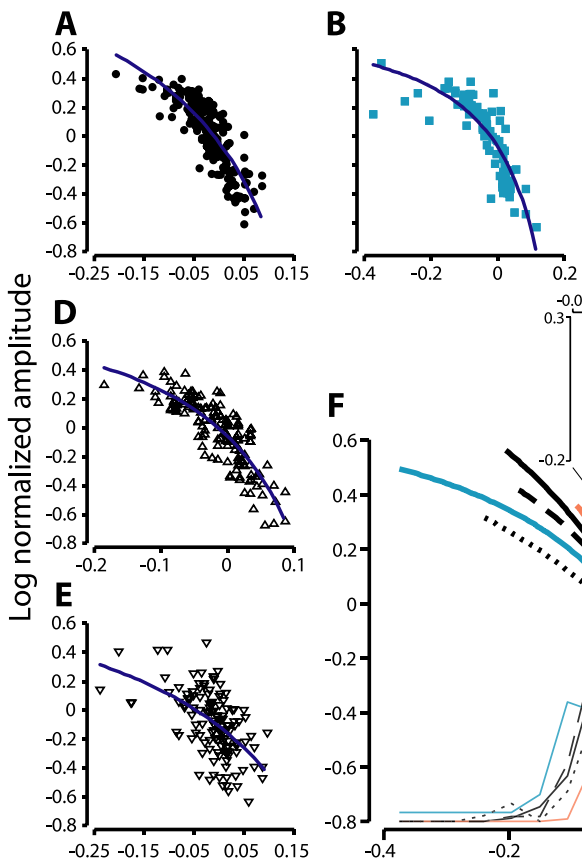

C

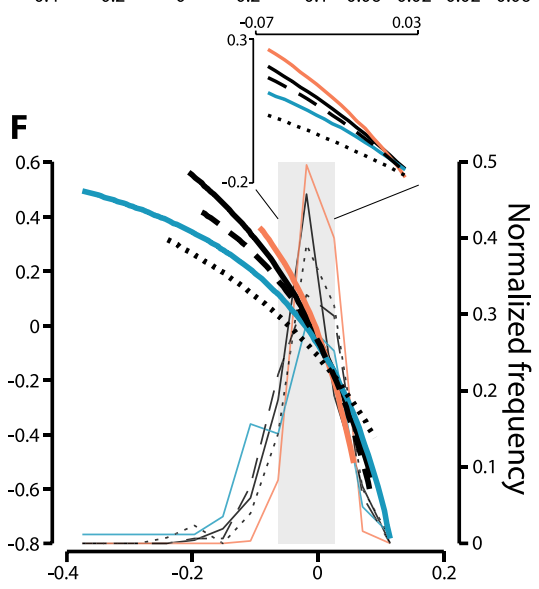

Log normalized latency

Figure 4. Relationship between saccade latency and amplitude for saccades evoked with different pulse patterns. $A-E$, Log normalized values of amplitude are plotted against log normalized values of latency for individual saccades at the three middling currents across all FEF sites separately for each pulse pattern: FIXED $(\boldsymbol{A})$, DECEL $(\boldsymbol{B})$, ACCEL $(\boldsymbol{C})$, RO $(\boldsymbol{D})$, and RI (E). Least-square fits are superimposed (dark blue lines). $\boldsymbol{F}$, The fits are replotted on the same axes with colors and line styles as in previous figures. In addition, histograms of saccade latency (right axis) are superimposed for each pulse pattern, normalized by the total number of saccades in that pulse pattern. The common range of latencies in which $\geq 95 \%$ of saccades occurred for each pulse pattern is denoted by gray shading. This range is enlarged in inset.

cades (or only for the averages). We regressed individual saccade amplitude on saccade latency separately for each pulse pattern, $\log$ normalized as above (Fig. 4A-E). We observed a strong inverse relationship whereby increasing latencies predicted decreasing amplitudes. These data were least-square fit with a saturation function (see Materials and Methods for expression) (parameter values are listed in Table S5, available at www.jneurosci.org as supplemental material) that explained between 61 and $72 \%$ of the variance in amplitude for each pulse pattern (with the exception of the random pattern, RI, 29\%).

Given that saccade amplitude was in part explained by saccade latency within a given pulse pattern, we were interested in whether the differences in amplitude between pulse patterns could be explained in part by differences in saccade latency between pulse patterns. The fits for individual pulse patterns are plotted together in Figure 4F. In particular, we focused on the range of latencies that were most common ( $\geq 95 \%$ of saccades) across pulse patterns (gray shaded region). That range is enlarged in the inset in Figure 4F. Note that the amplitude-to-latency relationship was very similar between pulse patterns, but there was a familiar ordering: ACCEL saccades were of largest amplitude, followed by FIXED, followed by DECEL when compared at a relatively short latency. The differences between pulse patterns were not maintained at longer latencies.

We have focused on the relationship between latency and amplitude, but the relationships of latency to peak velocity and latency to duration should also hold, as these metrics covary with amplitude (Becker, 1989). We confirmed that increased latencies predict slower peak velocities and shorter saccade durations for 
all pulse patterns (data not shown). In addition, for a given latency, the relative ordering of peak velocities as a function of pulse pattern was the same as for amplitude. However, latencymatched differences in saccade duration between pulse patterns were less clear.

In addition to comparing averages of saccade metrics, we were interested in the effect of pulse pattern on the trial-to-trial variability of saccadic vectors. We computed a measure of saccade scatter as the mean distance between the endpoint of individual saccades and their average endpoint for a given block of trials and pulse pattern (Dias and Segraves, 1999). We found a significant effect of saccade amplitude on scatter (ANCOVA, $F=49$, $\mathrm{df}=1$, $p<10^{-10}$ ), consistent with previous reports of saccades evoked from the superior colliculus (SC) (van Opstal and van Gisbergen, 1989 ) but found no effect of pulse pattern (ANCOVA, $p>0.98$ ) or saccade probability (ANCOVA, $p>0.87$ ).

\section{Saccade-triggered average of random pulse patterns}

The DECEL and ACCEL patterns were limited by their design to testing one class of pulse pattern, that of linearly and monotonically changing IPI. We implemented the two random patterns to sample the space of IPI orderings and IPI durations (while maintaining eight pulses in $35 \mathrm{~ms}$ ) and to measure their effects on saccades. For the RO pattern, the same IPIs used in the DECEL and ACCEL patterns were shuffled in an order that varied randomly from trial-to-trial. Thus, instantaneous frequencies were constant between the DECEL, ACCEL, and RO patterns, and only the order of those frequencies varied. Because each of the seven IPIs was used once per pulse train, the distribution of IPIs is flat (Fig. $5 A)$, with a mean of $5.00 \pm 0.04 \mathrm{~ms}( \pm \mathrm{SEM})$. For the RI pattern, we randomly assigned eight pulses to 2 -ms-wide time bins (one pulse per bin), resulting in seven IPIs ranging from 2 to $20 \mathrm{~ms}$. Because the chance of a pulse occurring at any remaining time bin was equal, the distribution of IPIs resembles an exponential probability distribution (Fig. $5 B$ ) as would be generated by a Poisson process and used to model physiological ISIs (for review, see Shadlen and Newsome, 1998). The mean IPI for the RI pattern was $4.16 \pm 0.06 \mathrm{~ms}( \pm \mathrm{SEM})$, slightly less than the other patterns, because RI trains could end before, but could not exceed, $35 \mathrm{~ms}$.

We analyzed the random pulse trains in search of a trend in pulse pattern that predicted train efficacy. Off-line, we segregated the random trains based on whether they evoked a saccade ("saccade trains") or failed to evoke a saccade ("null trains"). We computed a train profile as the average IPI at each of the seven interval positions. Computing this "train profile" separately for saccade trains and null trains produced a saccade-triggered average of IPI as a function of interval position (Fig. $5 D, E$ ). This differed from classical spike-triggered averaging in that we averaged with respect to an ordinal value of time (interval position) and not continuous time. We predicted that the saccadetriggered average of the most effective pulse trains would resemble the linear train profile of ACCEL, whereas the least effective trains would resemble the DECEL train profile (Fig. $5 C$ ). We tested this model by fitting a line to the set of all individual IPIs as a function of interval position separately for saccade and null trains. Consistent with our prediction, the saccade trains for both RO and RI were fit significantly with lines of negative slope (RO, $m=-0.062 \mathrm{~ms} /$ position, $r=-0.062, p<0.05$; RI, $m=-0.19$, $\left.r=-0.14, p<10^{-4}\right)$, whereas the null trains for both random patterns showed a linearly increasing trend of IPI with interval position (RO, $m=0.064, r=0.064, p<0.05$; RI, $m=0.13, r=$ $0.090, p<0.002$ ). The difference in slope between the saccade
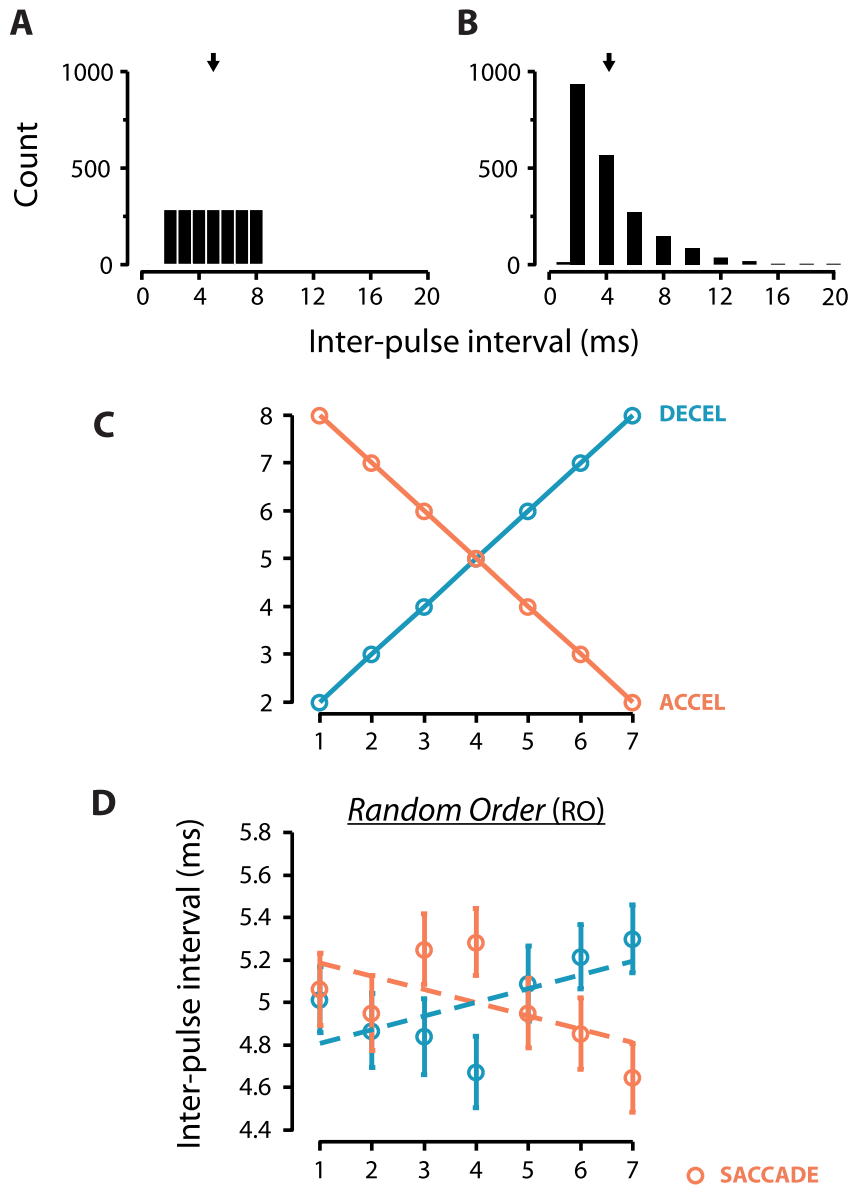

O SACCADE

E

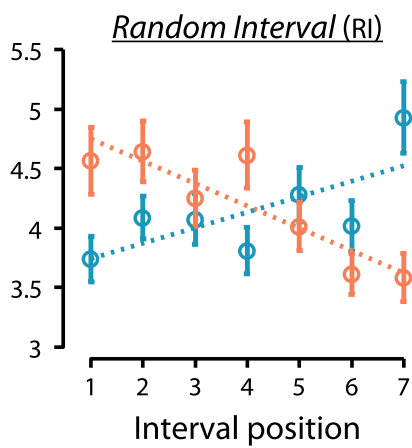

Figure 5. Saccade-triggered average of random pulse trains. $A, B$, Frequency distributions are shown for the IPIs drawn for Ro $(\boldsymbol{A})$ and RI $(\boldsymbol{B})$ across all trials, with downward arrows indicating the mean IPI. C, Train profiles (mean IPI vs interval position) for Accel (red circles and line) and DECEL (blue circles and line) represent our model for the most and least effective pulse patterns, respectively, and are provided for comparison to the random pulse patterns. $\boldsymbol{D}, \boldsymbol{E}$, Individual pulse trains from Ro $(\boldsymbol{D})$ and RI $(\boldsymbol{E})$ were stratified into saccade trains and null trains depending on whether they did or did not evoke a saccade, respectively. The train profiles for the saccade trains (red circles) and null trains (blue circles) are plotted. Error bars indicate SEM. Significant linear regressions $(p<0.05)$ of individual IPIs on interval position are superimposed on the train profiles for saccade trains (red lines) and, separately, for null trains (blue lines) from Ro (dashed lines) and RI (dotted lines). The distribution of mean IPIs for Ri is lower than that for ro because ei trains could end before, but could not exceed, the 35 ms duration imposed on all other patterns.

and null trails was significant for both random patterns (RO, $t=$ 2.84, $p<0.005$; RI, $t=5.14, p<10^{-6}$ ).

Importantly, the saccade and null trains did not differ in mean IPI collapsed across all interval positions (ANOVA: RO, $p=1$; RI, $p>0.68)$, nor did IPI change as a function of interval position 
when collapsing across saccade and null trains (ANOVA: RO, $p>$ 0.98; RI, $p>0.36$ ). In other words, the saccade trains did not, for example, have a lower mean IPI (higher frequency) than the null trains, nor did IPIs, for example, successively decrease (accelerating frequency) for all trains.

\section{Discussion}

Our results demonstrate that the saccadic system is sensitive to the temporal pattern of microstimulation independent of rate. ACCEL was three times more effective at evoking a saccade than the symmetrically opposite DECEL and also was more effective than FIXED. A saccade-triggered average of random pulse trains was consistent with the effective trains being, on average, of accelerating rate, whereas the ineffective trains were of decelerating rate. In addition to differences in efficacy, the saccade metrics differed as a function of pulse pattern. When controlled for efficacy, saccades evoked by DECEL occurred earlier and were larger, faster, and of longer duration than those of all other pulse patterns; the opposite was true for ACCEL.

The differences between ACCEL and DECEL are particularly interesting, because these patterns are mirror images of one another: identical intervals in exactly opposite order. Any differences in the efficacy of these two pulse patterns cannot be attributed to a specific instantaneous frequency or even a local average frequency across a subset of pulses. Rather, all differences must be attributed to the order of IPIs: a history dependence of stimulation frequency.

Recalling that higher stimulation frequencies are more effective at evoking a saccade (Tehovnik and Sommer, 1997), one possible explanation of the observed effect of pattern efficacy is that high frequencies are most effective when they occur later rather than earlier in the train (as in ACCEL), implying a priming effect of the early pulses. When the high-frequency pulses occur early in the train (as in DECEL), they do not benefit from this priming epoch and thus are less effective at evoking a saccade. The late low-frequency pulses in DECEL, although theoretically primed by the earlier high frequencies, are themselves much less effective at evoking a saccade (Glimcher and Sparks, 1993; Tehovnik and Sommer, 1997), thus accounting in part for the poorer efficacy of DECEL. An alternative explanation dismisses the notion of a priming effect and asserts that high-frequency pulses in both ACCEL and DECEL are sufficient on their own to evoke a saccade and that the poorer efficacy of DECEL is attributable to a suppressive effect of the later pulses. However, we know that very short stimulation trains ( $\leq 25 \mathrm{~ms}$ ) of comparable current and even greater frequency (up to $1000 \mathrm{~Hz}$ ) do not evoke saccades (Tehovnik and Sommer, 1997). Therefore, it is unlikely that the poorer efficacy of DECEL is explained by a suppressive effect of the later pulses. Instead, the high-frequency pulses are simply ineffective on their own; their efficacy depends on a preceding series of priming pulses.

In the present experiment, the priming pulses in ACCEL were always of a lower frequency than the final, saccade-triggering pulses. Therefore, we cannot conclude that lower frequencies are specially tuned for a priming function (in the way the high frequencies appear tuned for a saccade-evoking function), because priming pulses of other relative frequencies were not tested. However, we note the similarity between the ACCEL pulse pattern and the presaccadic spike trains observed physiologically: FEF neurons spike at low frequencies when preparing a saccade, followed by a burst of high-frequency firing immediately before saccade onset (Sparks, 1978; Bruce and Goldberg, 1985; Hanes and Schall, 1996). Moreover, activity at these lower frequencies is correlated with an increased probability of saccade (Basso and Wurtz, 1997; Dorris and Munoz, 1998), suggestive of a priming function. Second, the higher frequencies, when occurring early in the train, may have a suppressive effect on later pulses by recruiting long-lasting inhibition, as discussed below (Swadlow, 1992; Seidemann et al., 2002; Butovas and Schwarz, 2003; Butovas et al., 2006).

For purposes of interpretation, the above discussion implicitly reduces the ACCEL and DECEL patterns to binary epochs of fixed frequency: low-to-high and high-to-low, respectively. However, we recognize that the actual patterns consist of a continuously changing frequency and that this feature may contribute to the differential effects of the present pulse patterns (e.g., Tsodyks and Markram, 1997).

\section{Mechanism of pulse-pattern sensitivity}

A useful working model with which to consider a mechanism of pulse-pattern sensitivity is to assume that the sensitivity arises from differences in the number of spikes elicited within the oculomotor system (not necessarily the FEF), with more spikes leading to a greater probability of saccade. As such, pulse trains of accelerating frequency (e.g., ACCEL) are assumed to elicit more spikes than pulse trains of the same instantaneous frequencies only in decreasing order (e.g., DECEL). We consider three levels at which this differential effect of pulse pattern may occur.

\section{Differential direct activation}

Accelerating trains may more effectively elicit spikes in the neurons directly affected by the extracellular current flux of the microstimulation. For instance, very short IPIs falling within the neuronal refractory period may render portions of certain pulse trains ineffective. However, the shortest IPIs in DECEL are also present in ACCEL. Thus, by symmetry, any effects attributable to the refractory period would be equivalent between the two patterns, assuming that an intra-refractory period pulse does not influence the efficacy of subsequent pulses. Furthermore, microstimulation efficacy in macaque FEF is not degraded with IPIs as low as $1 \mathrm{~ms}$, twice as short as used presently (Tehovnik and Sommer, 1997).

\section{Differential synaptic transmission}

Accelerating trains may lead to greater postsynaptic depolarization. For instance, EPSPs may combine more efficiently for accelerating than decelerating inputs assuming a model of linear temporal summation and specific values of membrane properties. However, in previous work testing temporal input patterns in isolated Aplysia ganglia, Segundo et al. (1963) found that predictions based on linear temporal summation could not account for the effects of input pattern they observed (effects that were consistent with the trends observed here). The exact way in which temporal summation may contribute to discriminating pulse patterns is difficult to predict because nonlinearities in the summation of inputs (Margulis and Tang, 1998) and variation in the time window of integration, as short as $2 \mathrm{~ms}$ (Pouille and Scanziani, 2001), favor either ACCEL or DECEL or make no prediction depending on the instantaneous input frequency and the location of inputs on the target cell. In addition to temporal summation, synaptic depression could weaken transmission specifically of high-frequency signals, for instance, by depleting synaptic reserves (Tsodyks and Markram, 1997; Galarreta and Hestrin, 1998; Schneggenburger et al., 2002). This depression would occur relatively early in the course of a decelerating signal, thus impeding transmission of the remaining signal, while occurring later in an accelerating signal, and thus would be of less consequence. 
Differential dynamics of excitation and inhibition

Decelerating trains may recruit inhibition earlier in the train, thereby shunting later pulses. Converging experimental evidence suggests that the balance of excitation to inhibition in cortex is frequency dependent. As input frequency increases, activation of inhibitory interneurons rises more sharply than activation of excitatory neurons, and inhibitory synapses sustain if not facilitate transmission, whereas excitatory synapses depress (Galarreta and Hestrin, 1998; Reyes et al., 1998; Gibson et al., 1999; Tateno et al., 2004; Tateno and Robinson, 2006). In addition, other inhibitory neuromodulators are recruited disproportionately more at higher frequencies (e.g., Diana and Marty, 2004). Together, when high-frequency signals follow low-frequency signals (as in ACCEL), the inhibition is recruited after the input train has completed, and therefore the inhibition is of little immediate consequence. However, when high frequencies precede low frequencies (as in DECEL), the recruited inhibition coincides with the remaining excitatory input, thereby shunting these later signals and reducing the overall efficacy of the train.

Further supporting a feedback inhibition mechanism, in vivo microstimulation of macaque FEF (Seidemann et al., 2002) and rat barrel cortex (Butovas and Schwarz, 2003) causes a transient increase in spiking activity followed by a profound suppression for several hundred milliseconds. Similarly, in rabbit layer VI cortical neurons, subthreshold antidromic stimulation causes a brief decrease followed by long increase in spike threshold to adjacent extracellular stimulation (Swadlow, 1992). Evidence suggests the suppression of spiking activity is caused by recruitment of feedback inhibition rather than a decreased gain at excitatory synapses (Seidemann et al., 2002; Butovas and Schwarz, 2003; Butovas et al., 2006).

The frequency-dependent recruitment of shunting inhibition may account for the poor efficacy of DECEL relative to FIXED: not only are the early high frequencies unprimed, they may actively shunt the efficacy of later pulses. Furthermore, frequencydependent inhibition may explain why high-frequency microstimulation competing with an endogenous saccade plan will suppress saccades (unprimed trigger is both unsuccessful and shunts other inputs), whereas the same microstimulation applied in agreement with the intended saccade goal will facilitate saccades [endogenous plan primes exogenous trigger (Burman and Bruce, 1997; see also Schlag et al., 1998)]. In addition to protecting against unintended (unprimed) saccades, the suppression recruited by the high-frequency trigger may serve as an important resetting mechanism: inhibiting the FEF postsaccadically so that residual activity does not erroneously facilitate or average into subsequent saccades (Burman and Bruce, 1997; Seidemann et al., 2002).

The site of pulse-pattern sensitivity remains an open question. It may occur within the FEF, or the output neurons of the FEF may transmit the pulse-pattern signal faithfully to downstream oculomotor structures that in turn discriminate pulse pattern, such as the brainstem or SC (e.g., Segraves and Goldberg, 1987; Segraves, 1992; Hanes and Wurtz, 2001). Future studies might focus on the primary site of action of FEF microstimulation (local circuitry or output fibers) as well as the circuit response (excitatory or inhibitory) to individual microstimulation pulses within a train.

\section{Functional phases of saccadic signals}

Experiments in the FEF and SC suggest that the saccadegenerating signal consists of three phases. The first phase (referred to here as the "primer") is preparatory, with gradually
ACCEL

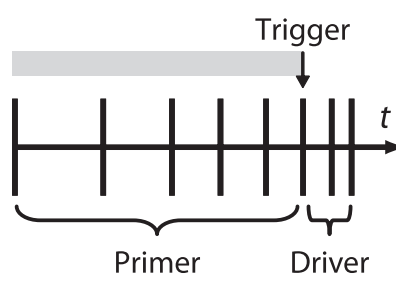

DECEL

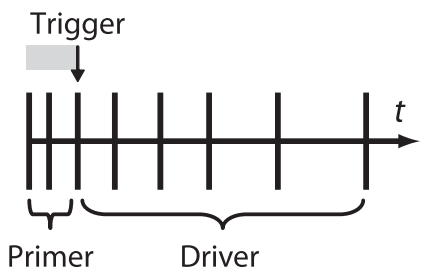

Figure 6. Primer-trigger-driver model of the saccade control signal. The pulse patterns for ACCEL (left) and DECEL (right) are depicted, and their proposed functional phases are labeled. Each vertical bar represents a biphasic microstimulation pulse; the horizontal lines represent time $(t)$ increasing from left to right. The saccade initiation signal (the "trigger"; black downward arrows) is associated with high-frequency pulses and thus occurs more toward the end of Accel but more toward the beginning of DECEL (the exact location of the trigger is approximate). Because the trigger initiates the saccade, a later trigger is associated with greater saccade latency, as represented by the length of the gray bar. The late trigger of ACCEL is consistent with ACCEL saccades demonstrating the longest latencies of all pulse patterns (DECEL saccades with their early trigger were of the shortest latency). Any pulses occurring before the trigger are termed the "primer," and those occurring after are termed the "driver." The primer is associated with preparing the movement and providing a pedestal of activity on which the trigger acts. Therefore, the greater the primer (in number of pulses and duration), the more likely the trigger will successfully evoke a saccade. The greater primer of ACCEL is consistent with ACCEL being the most effective pulse pattern (DECEL with its lesser primer was the least effective). The driver is associated with extending the eye movement once it has been initiated. The greater driver of DECEL is consistent with DECEL saccades commanding the largest amplitude, fastest peak velocity, and longest duration of all pulse patterns (the opposite was true for AcCEL with its lesser driver).

increasing or constant low-frequency activity specifying the saccadic vector (Glimcher and Sparks, 1992, 1993; Hanes et al., 1995). The second phase (the "trigger") initiates the saccade with a high-frequency burst of neuronal spiking activity (Sparks, 1978; Hanes et al., 1995). The third phase (the "driver") influences the movement itself, as evidenced by the finding that continued microstimulation after saccade onset (i.e., post-trigger) drives larger amplitude saccades (Stanford et al., 1996).

We propose a model in which portions of the presently used pulse trains are classified according to the above frequencydefined phases (Fig. 6). For instance, ACCEL and DECEL both have high-frequency, trigger-like portions: early in the train for DECEL but late for ACCEL. The relative position of the trigger within the train should correlate with saccade latency. We found that ACCEL saccades (late trigger) occurred with the longest latency, whereas DECEL saccades (early trigger) occurred with the shortest latency. This observation may be related to that of Hanes and Schall (1996), who showed that voluntary saccade latency was positively correlated with the time to reach a high-frequency rate threshold.

The pretrigger pulses form the primer, which is of greater magnitude (more pulses and of longer duration) for ACCEL than DECEL. The primer should facilitate saccades by providing a pedestal of activity with which the trigger can combine to reach some threshold. Experiments in the SC show that increased lowfrequency, presaccadic activity predicts an increased probability of a saccade toward the preferred direction of the active neurons (Basso and Wurtz, 1997; Dorris and Munoz, 1998) and microstimulation of the SC and FEF that is itself too weak to evoke saccades biases the subject's choices toward targets in the movement field of the stimulated site (Glimcher and Sparks, 1993; Schiller and Tehovnik, 2001). It follows that the greater the primer, the greater the probability of saccade. This model is consistent with our results: ACCEL was the most effective, whereas DECEL was least effective. Furthermore, the random patterns were of intermediate efficacy, which is consistent with the fact that the average of either random pattern resembles the average of DECEL 
and ACCEL; the random patterns also were less sensitive to changes in stimulation current (Fig. 2A), which is consistent with a greater trial-to-trial variability in primer magnitude.

The driver is composed of the post-trigger pulses and is of greater magnitude for DECEL than ACCEL. The driver should contribute to saccade amplitude, peak velocity, and duration, all of which are correlated for voluntary (Becker, 1989) and stimulation-evoked saccades (Chen, 2006; Knight and Fuchs, 2006; our unpublished observations). We found that DECEL saccades (greater driver), although less likely to occur, were of the greatest amplitude, peak velocity, and duration. The opposite was true for ACCEL saccades (lesser driver).

We reasoned that if the differences in saccade metrics between pulse patterns could be explained in part by systematic differences in trigger timing and driver duration, then perhaps the trial-to-trial variation in the exact timing of these signals could explain variation in saccade metrics within a pulse pattern. The strong inverse relationship between saccade latency (an approximation of trigger timing) and amplitude for each pulse pattern (Fig. 4) is consistent with this reasoning and supports the primertrigger-driver model. Specifically, the latency-amplitude relationship suggests that the temporal properties of the trigger and driver are interdependent even within an identical pulse pattern (as the model predicts), not merely between classes pulse patterns, for which some other systematic difference may explain pattern-dependent differences in saccade metrics.

The similarity of the latency-amplitude relationship across pulse patterns (Fig. $4 F$ ) suggests that systematic differences in trigger timing (and consequently driver duration) between pulse patterns accounts in part for the pattern-dependent differences in average amplitude. However, the slight differences in amplitude between pulse patterns for a given latency (Fig. $4 F$, inset) suggest that factors other than trigger timing and driver duration may also contribute to saccade amplitude. In examining the temporal structure of each pulse pattern (Fig. $1 A$ ), one notes that for identical trigger times, the ACCEL train will have more driver pulses than FIXED, which will in turn have more than DECEL. Given the dependence of saccade amplitude on the number of microstimulation pulses independent of their frequency (Stanford et al., 1996), this difference in the number of driver pulses between pulse patterns for a given trigger time predicts that, at a given latency, saccades evoked with ACCEL should be of larger amplitude than those of FIXED, which in turn should be larger than those of DECEL. We observed this trend for saccades of relatively short latencies and therefore sufficiently long drivers to realize differences in the number of pulses (Fig. $4 F$, inset). For longer latencies, pattern-dependent differences in the number of driver pulses are reduced (at the limit, a trigger on the last pulse will have zero driver pulses regardless of pulse pattern), and therefore one predicts the amplitudes to converge across pulse patterns, as was observed.

The ability of low frequencies to bias direction but not triggering of saccades (Glimcher and Sparks, 1993; Schiller and Tehovnik, 2001), the tendency of high frequencies to trigger saccades (Sparks, 1978; Hanes et al., 1995), and the relationship between the number (not rate) of post-trigger pulses and amplitude (Stanford et al., 1996) imply that the frequency of a particular epoch within the saccadic signal serves to define the functional role of that epoch (primer or trigger, with driver following the trigger) rather than modulate the strength of a role defined by some other means. Frequency may therefore provide a mechanism to multiplex several functional signals over the same population of neurons.

\section{Implications for clinical applications and neural coding}

Electrical stimulation of the CNS, in the form of DBS, plays an increasingly broad role in the treatment of neurological and psychiatric disorders (Perlmutter and Mink, 2006). Despite its therapeutic efficacy, little is known about how the exact stimulation parameters account for functional outcomes and how protocols could be refined for maximal clinical benefit (Lozano et al., 2002; Moro et al., 2002; Butson et al., 2007). One limitation in optimizing stimulation parameters is the amount of current tolerated by the patient. In general, increasing the current increases the desired therapeutic effect but also amplifies undesirable side effects attributable to passive current spread (Rizzone et al., 2001), tissue damage (Tehovnik, 1996), and need for repeated surgeries to replace the stimulation battery (Joint et al., 2002). The present study shows that by manipulating pulse pattern, one can achieve identical efficacy with less stimulation current. Pulse pattern could therefore be used clinically to maximize therapeutic effect while minimizing side effects. The pulse patterns used presently are defined in finite time windows and are of dynamic rate, whereas DBS trains are typically chronic (e.g., Limousin et al., 1998). However, early experiments on peripheral nerves provide a proof-of-principle that effective, short pulse patterns can be concatenated to form a repeating, continuous train that is still more effective than its fixed IPI counterpart (Ripley and Wiersma, 1953).

As emphasized above, the stimulation rate of the pulse patterns was identical when averaged across the entire $35 \mathrm{~ms}$ of each train (the RI pattern excepted, as discussed). Therefore, for the oculomotor system to discriminate the pulse patterns using average rate, the time window of integration $(d t)$ must be $<35 \mathrm{~ms}$. However, a narrower $d t$ by itself cannot discriminate ACCEL from DECEL, because their local averages are identical for all values of $d t$. Instead, the system must additionally be sensitive to the order of local averages (low $\rightarrow$ high vs high $\rightarrow$ low), a form of temporal coding. It is possible that the oculomotor system exploits this sensitivity to temporal pattern in the generation of voluntary saccades. Consistent with this possibility is the similarity between the accelerating rate of the most effective pulse pattern (ACCEL) and the prototypical build-up of endogenous activity preceding a saccade (Bruce and Goldberg, 1985; Munoz and Wurtz, 1995). Therefore, the efficacy of the ACCEL pattern may arise from emulating an endogenous signal that is itself optimized. Indeed, past microstimulation studies have mimicked this endogenous signal and used an accelerating pulse pattern (McPeek et al., 2003; McPeek, 2006). Given the controversy as to the importance of precise spike timing to neural coding (e.g., Softky, 1995; de Ruyter van Steveninck et al., 1997; Shadlen and Newsome, 1998; Hopfield and Brody, 2000), patterned microstimulation may provide a useful tool for testing the causal role of temporal information in neural circuits.

\section{References}

Afraz SR, Kiani R, Esteky H (2006) Microstimulation of inferotemporal cortex influences face categorization. Nature 442:692-695.

Basso MA, Wurtz RH (1997) Modulation of neuronal activity by target uncertainty. Nature 389:66-69.

Becker W (1989) Metrics. In: Reviews of oculomotor research, Vol 3, The neurobiology of saccadic eye movements (Wurtz RH, Goldberg ME, eds), pp 13-68. New York: Elsevier.

Brecht M, Singer W, Engel AK (2004) Amplitude and direction of saccadic eye movements depend on the synchronicity of collicular population activity. J Neurophysiol 92:424-432.

Brindley GS, Lewin WS (1968) The sensations produced by electrical stimulation of the visual cortex. J Physiol (Lond) 196:479-493. 
Bruce CJ, Goldberg ME (1985) Primate frontal eye fields. I. Single neurons discharging before saccades. J Neurophysiol 53:603-635.

Bruce CJ, Goldberg ME, Bushnell MC, Stanton GB (1985) Primate frontal eye fields. II. Physiological and anatomical correlates of electrically evoked eye movements. J Neurophysiol 54:714-734.

Burman DD, Bruce CJ (1997) Suppression of task-related saccades by electrical stimulation in the primate's frontal eye field. J Neurophysiol 77:2252-2267.

Butovas S, Schwarz C (2003) Spatiotemporal effects of microstimulation in rat neocortex: a parametric study using multielectrode recordings. J Neurophysiol 90:3024-3039.

Butovas S, Hormuzdi SG, Monyer H, Schwarz C (2006) Effects of electrically coupled inhibitory networks on local neuronal responses to intracortical microstimulation. J Neurophysiol 96:1227-1236.

Butson CR, Cooper SE, Henderson JM, McIntyre CC (2007) Patientspecific analysis of the volume of tissue activated during deep brain stimulation. Neuroimage 34:661-670.

Cavanaugh J, Wurtz RH (2004) Subcortical modulation of attention counters change blindness. J Neurosci 24:11236-11243.

Chen LL (2006) Head movements evoked by electrical stimulation in the frontal eye field of the monkey: evidence for independent eye and head control. J Neurophysiol 95:3528-3542.

de Ruyter van Steveninck RR, Lewen GD, Strong SP, Koberle R, Bialek W (1997) Reproducibility and variability in neural spike trains. Science 275:1805-1808.

Diana MA, Marty A (2004) Endocannabinoid-mediated short-term synaptic plasticity: depolarization-induced suppression of inhibition (DSI) and depolarization-induced suppression of excitation (DSE). Br J Pharmacol 142:9-19.

Dias EC, Segraves MA (1999) Muscimol-induced inactivation of monkey frontal eye field: effects on visually and memory-guided saccades. J Neurophysiol 81:2191-2214.

Dorris MC, Munoz DP (1998) Saccadic probability influences motor preparation signals and time to saccadic initiation. J Neurosci 18:7015-7026.

Ferrier D (1886) The functions of the brain, Ed 2. New York: G. P. Putnam's Sons.

Fritsch G, Hitzig E (1870) On the electrical excitability of the cerebrum (in German). Arch f Anat, Physiol und wissenschaftl Mediz 37:300-332.

Galarreta M, Hestrin S (1998) Frequency-dependent synaptic depression and the balance of excitation and inhibition in the neocortex. Nat Neurosci 1:587-594.

Gibson JR, Beierlein M, Connors BW (1999) Two networks of electrically coupled inhibitory neurons in neocortex. Nature 402:75-79.

Glimcher PW, Sparks DL (1992) Movement selection in advance of action in the superior colliculus. Nature 355:542-545.

Glimcher PW, Sparks DL (1993) Effects of low-frequency stimulation of the superior colliculus on spontaneous and visually guided saccades. J Neurophysiol 69:953-964.

Gold JI, Shadlen MN (2003) The influence of behavioral context on the representation of a perceptual decision in developing oculomotor commands. J Neurosci 23:632-651.

Graziano MS, Taylor CS, Moore T (2002) Complex movements evoked by microstimulation of precentral cortex. Neuron 34:841-851.

Hanes DP, Schall JD (1996) Neural control of voluntary movement initiation. Science 274:427-430.

Hanes DP, Wurtz RH (2001) Interaction of the frontal eye field and superior colliculus for saccade generation. J Neurophysiol 85:804-815.

Hanes DP, Thompson KG, Schall JD (1995) Relationship of presaccadic activity in frontal eye field and supplementary eye field to saccade initiation in macaque: Poisson spike train analysis. Exp Brain Res 103:85-96.

Hanks TD, Ditterich J, Shadlen MN (2006) Microstimulation of macaque area LIP affects decision-making in a motion discrimination task. Nat Neurosci 9:682-689.

Hopfield JJ, Brody CD (2000) What is a moment? "Cortical" sensory integration over a brief interval. Proc Natl Acad Sci USA 97:13919-13924.

Joint C, Nandi D, Parkin S, Gregory R, Aziz T (2002) Hardware-related problems of deep brain stimulation. Mov Disord 17 [Suppl 3]:S175-S180.

Knight TA, Fuchs AF (2006) Contribution of the frontal eye field to gaze shifts in the head-unrestrained monkey: effects of microstimulation. J Neurophysiol 97:618-634.

Limousin P, Krack P, Pollak P, Benazzouz A, Ardouin C, Hoffmann D, Bena- bid AL (1998) Electrical stimulation of the subthalamic nucleus in advanced Parkinson's disease. N Engl J Med 339:1105-1111.

Lozano AM, Dostrovsky J, Chen R, Ashby P (2002) Deep brain stimulation for Parkinson's disease: disrupting the disruption. Lancet Neurol 1:225-231.

Margulis M, Tang CM (1998) Temporal integration can readily switch between sublinear and supralinear summation. J Neurophysiol 79:2809-2813.

McPeek RM (2006) Incomplete suppression of distractor-related activity in the frontal eye field results in curved saccades. J Neurophysiol 96:2699-2711.

McPeek RM, Han JH, Keller EL (2003) Competition between saccade goals in the superior colliculus produces saccade curvature. J Neurophysiol 89:2577-2590.

Moore T, Armstrong KM (2003) Selective gating of visual signals by microstimulation of frontal cortex. Nature 421:370-373.

Moore T, Fallah M (2001) Control of eye movements and spatial attention. Proc Natl Acad Sci USA 98:1273-1276.

Moore T, Fallah M (2004) Microstimulation of the frontal eye field and its effects on covert spatial attention. J Neurophysiol 91:152-162.

Moro E, Esselink RJ, Xie J, Hommel M, Benabid AL, Pollak P (2002) The impact on Parkinson's disease of electrical parameter settings in STN stimulation. Neurology 59:706-713.

Muller JR, Philiastides MG, Newsome WT (2005) Microstimulation of the superior colliculus focuses attention without moving the eyes. Proc Natl Acad Sci USA 102:524-529.

Munoz DP, Wurtz RH (1995) Saccade-related activity in monkey superior colliculus. I. Characteristics of burst and buildup cells. J Neurophysiol 73:2313-2333.

Murasugi CM, Salzman CD, Newsome WT (1993) Microstimulation in visual area MT: effects of varying pulse amplitude and frequency. J Neurosci 13:1719-1729.

Penfield W, Jasper HH (1954) Epilepsy and the functional anatomy of the human brain, Ed 1. Boston: Little.

Perlmutter JS, Mink JW (2006) Deep brain stimulation. Annu Rev Neurosci 29:229-257.

Pouille F, Scanziani M (2001) Enforcement of temporal fidelity in pyramidal cells by somatic feed-forward inhibition. Science 293:1159-1163.

Reyes A, Lujan R, Rozov A, Burnashev N, Somogyi P, Sakmann B (1998) Target-cell-specific facilitation and depression in neocortical circuits. Nat Neurosci 1:279-285.

Ripley SH, Wiersma CAG (1953) The effect of space stimulation of excitatory and and inhibitory axons in the crayfish. Physiol Comp Ocol Int J Comp Physiol Ecol 3:1-17.

Rizzone M, Lanotte M, Bergamasco B, Tavella A, Torre E, Faccani G, Melcarne A, Lopiano L (2001) Deep brain stimulation of the subthalamic nucleus in Parkinson's disease: effects of variation in stimulation parameters. J Neurol Neurosurg Psychiatry 71:215-219.

Robinson DA (1972) Eye movements evoked by collicular stimulation in the alert monkey. Vision Res 12:1795-1808.

Robinson DA, Fuchs AF (1969) Eye movements evoked by stimulation of frontal eye fields. J Neurophysiol 32:637-648.

Rolls ET (1975) The neural basis of brain-stimulation reward. Prog Neurobiol 3:73-160.

Romo R, Hernandez A, Zainos A, Salinas E (1998) Somatosensory discrimination based on cortical microstimulation. Nature 392:387-390.

Russo GS, Bruce CJ (1993) Effect of eye position within the orbit on electrically elicited saccadic eye movements: a comparison of the macaque monkey's frontal and supplementary eye fields. J Neurophysiol 69:800-818.

Salzman CD, Britten KH, Newsome WT (1990) Cortical microstimulation influences perceptual judgements of motion direction. Nature 346:174-177.

Schiller PH, Tehovnik EJ (2001) Look and see: how the brain moves your eyes about. Prog Brain Res 134:127-142.

Schlag J, Dassonville P, Schlag-Rey M (1998) Interaction of the two frontal eye fields before saccade onset. J Neurophysiol 79:64-72.

Schneggenburger R, Sakaba T, Neher E (2002) Vesicle pools and short-term synaptic depression: lessons from a large synapse. Trends Neurosci 25:206-212.

Segraves MA (1992) Activity of monkey frontal eye field neurons projecting to oculomotor regions of the pons. J Neurophysiol 68:1967-1985. 
Segraves MA, Goldberg ME (1987) Functional properties of corticotectal neurons in the monkey's frontal eye field. J Neurophysiol 58:1387-1419.

Segundo JP, Moore GP, Stensaas LJ, Bullock TH (1963) Sensitivity of neurones in Aplysia to temporal pattern of arriving impulses. J Exp Biol 40:643-667.

Seidemann E, Arieli A, Grinvald A, Slovin H (2002) Dynamics of depolarization and hyperpolarization in the frontal cortex and saccade goal. Science 295:862-865.

Shadlen MN, Newsome WT (1998) The variable discharge of cortical neurons: implications for connectivity, computation, and information coding. J Neurosci 18:3870-3896.

Softky WR (1995) Simple codes versus efficient codes. Curr Opin Neurobiol 5:239-247.

Sparks DL (1978) Functional properties of neurons in the monkey superior colliculus: coupling of neuronal activity and saccade onset. Brain Res 156:1-16.

Sparks DL, Mays LE, Porter JD (1987) Eye movements induced by pontine stimulation: interaction with visually triggered saccades. J Neurophysiol 58:300-318.

Stanford TR, Freedman EG, Sparks DL (1996) Site and parameters of microstimulation: evidence for independent effects on the properties of saccades evoked from the primate superior colliculus. J Neurophysiol 76:3360-3381.

Strick PL (2002) Stimulating research on motor cortex. Nat Neurosci 5:714-715.

Swadlow HA (1992) Monitoring the excitability of neocortical efferent neu- rons to direct activation by extracellular current pulses. J Neurophysiol 68:605-619.

Tateno T, Robinson HP (2006) Rate coding and spike-time variability in cortical neurons with two types of threshold dynamics. J Neurophysio 95:2650-2663.

Tateno T, Harsch A, Robinson HP (2004) Threshold firing frequencycurrent relationships of neurons in rat somatosensory cortex: type 1 and type 2 dynamics. J Neurophysiol 92:2283-2294.

Tehovnik EJ (1996) Electrical stimulation of neural tissue to evoke behavioral responses. J Neurosci Methods 65:1-17.

Tehovnik EJ, Lee K (1993) The dorsomedial frontal cortex of the rhesus monkey: topographic representation of saccades evoked by electrical stimulation. Exp Brain Res 96:430-442.

Tehovnik EJ, Sommer MA (1997) Electrically evoked saccades from the dorsomedial frontal cortex and frontal eye fields: a parametric evaluation reveals differences between areas. Exp Brain Res 117:369-378.

Tolias AS, Sultan F, Augath M, Oeltermann A, Tehovnik EJ, Schiller PH, Logothetis NK (2005) Mapping cortical activity elicited with electrical microstimulation using FMRI in the macaque. Neuron 48:901-911.

Tsodyks MV, Markram H (1997) The neural code between neocortical pyramidal neurons depends on neurotransmitter release probability. Proc Natl Acad Sci USA 94:719-723.

van Opstal AJ, van Gisbergen JA (1989) Scatter in the metrics of saccades and properties of the collicular motor map. Vision Res 29:1183-1196.

Williams ZM, Eskandar EN (2006) Selective enhancement of associative learning by microstimulation of the anterior caudate. Nat Neurosci 9:562-568. 